\title{
Presentation of Neuromyelitis Optica with Recurrent Severe Myelitis and Acute Respiratory Failure in an Old Woman
}

\author{
Saeed Razmeh, ${ }^{1, *}$ Zahra Ghurchian, ${ }^{1}$ Amir Hasan Habibi, ${ }^{1}$ Farzad Sina, ${ }^{1}$ and Mostafa Almasi ${ }^{1}$ \\ ${ }^{1}$ Neurology Department, Rasoul Akram Hospital, Iran University of Medical Sciences, Tehran, Iran \\ "Corresponding author: Saeed Razmeh, First unit, Number 57, West 17th St, Ghalam St, Shahinvilla, Tehran, Iran. Tel: +98-9397631901, E-mail: srazme82@gmail.com
}

Received 2017 June 03; Revised 2017 March 07; Accepted 2017 June 09.

\begin{abstract}
Neuromyelitis Optica (NMO) is a rare disease of the central nervous system that causes optic nerve and spinal cord involvement. The our patient first developed acute paraplegia that was treated with intravenous methylprednisolone with diagnosis of acute thoracic myelitis according to magnetic resonance imaging (MRI) finding, concurrently with tapering of oral prednisolone, again affected by quadriplegia and respiratory failure. She was seropositivity for NMO-IgG that was negative in first admission and MRI of spine shows hyperintense lesion in whole cervical and upper thoracic MRI. With considering the findings, NMO was diagnosed and the plasmapheresis starts for her. We report a case of this syndrome because it can increase the physician's awareness of the unusual manifestations of this syndrome.
\end{abstract}

Keywords: Neuromyelitis Optica, Myelitis, Respiratory Failure

\section{Introduction}

NMO, also called Devic disease, is an uncommon form of inflammatory demyelinating diseases of the central nervous system (CNS) that cause optic neuritis and transverse myelitis [1]. In other words, it is an autoimmune inflammatory disease of the CNS in which NMO-IgG binds to the aquaporin-4 (AQP4) water channel. it is may be confused with multiple sclerosis but it has distinctive clinical and pathological features, mainly it causes necrosis of the spinal cord and affecting white matter and gray matter of the brain and spinal cord and usually with an absence of OCB in cerebrospinal fluid (CSF) [2-4]. Anti-aquaporin 4 antibody (NMO IgG $\mathrm{AB}$ ) is the useful test that is highly specific and sensitive. The treatment for this disease involves acute management of acute attacks with intravenous (IV) methylprednisolone and plasma exchange, and prevention of future attacks with immunosuppressants including mycophenolate mofetil, azathioprine and rituximab $[5,6]$. We report a severe seropositive NMO in old Iranian woman with recurrent severe myelitis and respiratory failure.

\section{Case Presentation}

A73-year-old lady who was referred to our hospital with 7 days history of lower limbs weakness, gait disturbance and urinary retention that progress to paraplegia during 2 days. She had no history of medical problems, doesn't take any medication and her family history, social history, and review of systems were negative. On physical examination she was conscious and had stable vital signs, he had no neck vein engorgement, no carotid bruit. Chest examination was normal. She had, normal rate and regular rhythm, no murmur and without respiratory distress. Abdomen was soft, non-tender, without hepatosplenomegaly. In the extremities the pulses were good and symmetric. The neurological examination showed paraplegia with hypotonia, hyporeflexia and bilateral extensor plantar response with T6 level. Basic blood test shows hyperglycemia. B12 level was normal. HIV, HTLV1 and both vasculitis and malignancy workup were negative. NMO antibody was positive. Brain and whole spine MRI show hyperintensity in periventricular, juxtacortical area with a hyperintense longitudinal lesion in $\mathrm{T} 2$ weighted sequences of the thoracic spinal cord. Cerebrospinal fluid (CSF) was normal in cell count, protein and glucose. Viral polymerase chain reaction (PCR) and Oligoclonal bands (OCB) were negative. The patient was treated with a 5 -day course of intravenous methylprednisolone (1gr daily) with diagnosis of NMO that achieved mild improvement. She was discharged on oral prednisolone and folic acid and mineral. Concurrently with the tapering of prednisolone, again approximately 2 monthly later, the patient presented to emergency room with quadriplegia, dyspnea and bladder and bowel dysfunction. MRI of spine shows hyperintense lesion in whole cervical and upper thoracic MRI (Figure 1). Plasma exchange was started for patient with total dose $250 \mathrm{~mL} / \mathrm{kg}$ but in 4 days of admission, she developed respiratory arrest and intubated with mechanical ventilation in ICU and 
ultimately 2 weeks later expired due to cardiac arrest.

\section{Figure 1. Thoracic MRI of First Admission}

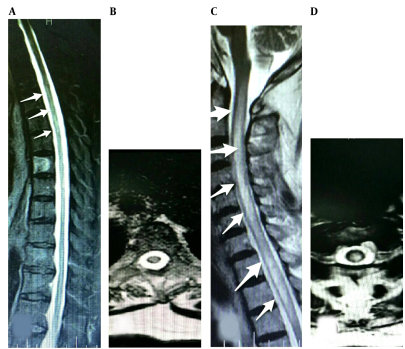

A, sagital T2 MRI showing hyperintense longitudinal in thoracic spine. B, Axial T2 MRI showing hyperintense intramedullary lesion whit white matter and gray matter involvement. Cervicothoracic MRI of second admission (C and D); C, Sagital hyperintense longitudinal lesion in cervical and upper thoracic spine, with expansion of cord. D, Axial T2 MRI showing hyperintense intramedullary lesion.

\section{Discussion}

NMO characterized by involvement of optic nerve and spinal cord [7]. The pathogenesis of NMO is mediated by the humoral immune system in contrast to the cellular mechanism that is proposed for MS, NMO-IgG (anti-AQP4) play a direct role in the pathogenesis of NMO. Aquaporin-4 (AQP4), is a water channel protein highly concentrated in spinal cord gray matter surrounding the central canal, periaqueductal and Virchow-robin space in the periventricular regions, and astrocytic foot processes at the blood-brain barrier [8]. The patient that seropositive for this antibody, are at risk of relapse but in general the recurrence rate in patient with longitudinal extensive transverse myelitis (LETM) is uncertain and continue to be a subject of case reports [9]. Our patient also has the recurrent severe myelitis during 2 months that is unusual. Furthermore, the median age for presentation of NMO is $32-42$ years, although cases present in older and young population, but the presentation at this age usually uncommon and until this time, our case is the oldest reported case in Iran. The CSF has pleocytosis in $80 \%$ - $85 \%$ patients of Devic disease although in our case was normal. Another interesting point in our patient is sudden onset of respiratory arrest without evidence of pneumonia or pulmonary embolism that may be due to upper cervical or medullary involvement in the acute phase. The plasma exchange recommended for moderate to severe transverse myelitis and optic neuritis and if refractory to prednisolone and plasma exchange, immunosuppression with cyclophosphamide recommended and due to high risk of recurrence, maintenance therapy with Azathioprine, rituximab, mycophenolate mofetil, methotrexate, prednisone, or mitoxantrone should be considered and at first Azathioprine and prednisone was prescribed that if don't respond to this drug it is necessary to change to other drugs [10]. Recently, many studies suggest that the therapeutic option in NMO should be immunosuppressive rather than immunomodulatory drugs [11]. In study Mok and his colleagues showed that use of cyclophosphamide was successful in halting relapses in a patient with systemic lupus erythematosus-associated NMO who was unresponsive to high-dose oral and intravenous corticosteroids, intravenous immunoglobulin, mycophenolate mofetil, tacrolimus, low-dose daily oral cyclophosphamide and rituximab [12]. Unfortunately our case doesn't respond to treatment and during plasma exchange was expired.

\subsection{Conclusion}

NMO can cause respiratory failure with involvement of upper cervical spinal cord and brain stem that require rapid diagnosis and aggressive treatment to prevent disease progression, also Devic disease requires Cytotoxic drugs and close follow-up after discharge to prevent relapse which can be debilitating and fatal.

\section{Acknowledgments}

We thank Mr, Majid Kheradmand for technical and editorial preparation of the manuscript.

\section{References}

1. Pearce JM. Neuromyelitis optica. Spinal Cord. 2005;43(11):631-4. doi: 10.1038/sj.sc.3101758. [PubMed: 15968305].

2. Lucchinetti CF, Mandler RN, McGavern D, Bruck W, Gleich G, Ransohoff RM, et al. A role for humoral mechanisms in the pathogenesis of Devic's neuromyelitis optica. Brain. 2002;125(Pt 7):1450-61. doi: 10.1093/brain/awf151. [PubMed: 12076996].

3. Lennon VA, Wingerchuk DM, Kryzer TJ, Pittock SJ, Lucchinetti CF, Fujihara $\mathrm{K}$, et al. A serum autoantibody marker of neuromyelitis optica: distinction from multiple sclerosis. Lancet. 2004;364(9451):2106-12. doi: 10.1016/S0140-6736(04)17551-X. [PubMed: 15589308].

4. Wingerchuk DM, Weinshenker BG. Neuromyelitis optica. Curr Treat Options Neurol. 2008;10(1):55-66. doi: 10.1007/s11940-008-0007-z. [PubMed: 18325300].

5. McKeon A, Fryer JP, Apiwattanakul M, Lennon VA, Hinson SR, Kryzer TJ, et al. Diagnosis of neuromyelitis spectrum disorders: comparative sensitivities and specificities of immunohistochemical and immunoprecipitation assays. Arch Neurol. 2009;66(9):1134-8. doi:10.1001/archneurol.2009.178. [PubMed: 19752303].

6. Waters PJ, McKeon A, Leite MI, Rajasekharan S, Lennon VA, Villalobos A, et al. Serologic diagnosis of NMO: a multicenter comparison of aquaporin-4-IgG assays. Neurology. 2012;78(9):665-71. doi: 10.1212/WNL.0b013e318248dec1. [PubMed: 22302543] discussion 669.

7. Sellner J, Boggild M, Clanet M, Hintzen RQ, Illes Z, Montalban $\mathrm{X}$, et al. EFNS guidelines on diagnosis and management of neuromyelitis optica. Eur J Neurol. 2010;17(8):1019-32. doi: 10.1111/j.14681331.2010.03066.x. [PubMed: 20528913]. 
8. Bennett JL, Lam C, Kalluri SR, Saikali P, Bautista K, Dupree C, et al. Intrathecal pathogenic anti-aquaporin-4 antibodies in early neuromyelitis optica. Ann Neurol. 2009;66(5):617-29. doi: 10.1002/ana.21802. [PubMed: 19938104].

9. Jung JS, Bhat RV, Preston GM, Guggino WB, Baraban JM, Agre P. Molecular characterization of an aquaporin cDNA from brain: candidate osmoreceptor and regulator of water balance. Proc Natl Acad Sci U S A. 1994;91(26):13052-6. doi: 10.1073/pnas.91.26.13052. [PubMed: 7528931].

10. Kimbrough DJ, Fujihara K, Jacob A, Lana-Peixoto MA, Leite MI,
Levy M, et al. Treatment of Neuromyelitis Optica: Review and Recommendations. Mult Scler Relat Disord. 2012;1(4):180-7. doi: 10.1016/j.msard.2012.06.002. [PubMed: 24555176].

11. Collongues N, de Seze J. Current and future treatment approaches for neuromyelitis optica. Ther Adv Neurol Disord. 2011;4(2):111-21. doi: 10.1177/1756285611398939. [PubMed: 21694808].

12. Mok CC, To $\mathrm{CH}$, Mak A, Poon WL. Immunoablative cyclophosphamide for refractory lupus-related neuromyelitis optica.J Rheumatol. 2008;35(1):172-4. [PubMed: 18176991]. 